\title{
Transgender and Nontrans Patients Do Not Receive Statistically Different Quality Primary Care at Whitman-Walker Health, 2008-2016
}

\author{
Deborah A. Goldstein, ${ }^{1,2, *}$ Eleanor Sarkodie, ${ }^{1}$ and W. David Hardy ${ }^{3}$
}

\begin{abstract}
Purpose: Washington, DC, has the highest prevalence of transgender persons in the United States at 2.8\%. Transgender persons in DC have lower income, less stable housing, and more HIV infection than nontrans persons. Data are scarce regarding primary care quality among trans persons. We provide a detailed analysis of transgender patients at Whitman-Walker Health, an HIV- and LGBT-focused community health center.

Methods: We performed a retrospective electronic medical record review of transgender patients $\geq 18$ years of age from 2008 to 2016, evaluating demographic factors, HIV status, gender-affirming care, and primary care quality indicators.

Results: Of 20,097 patients, 1822 (9.0\%) self-identify as transgender (62.9\% trans female and 37.2\% trans male), and 18,275 were nontransgender. Transgender patients are more likely to be young, white, HIV negative, and reside outside Washington, DC, than nontrans patients. Transgender patients are more likely to engage in primary care and have a similar likelihood of mammogram and colonoscopy screening than nontrans patients. Trans males are more likely to be privately insured, have lower rates of HIV testing than nontrans patients, and have higher rates of cervical Pap smears than cis females. Trans females have a high prevalence of HIV infection (26.6\%). Conclusion: This is the largest single-center U.S. transgender cohort to date. Over a quarter of trans females are HIV positive, consistent with a national prevalence of $27.7 \%$. Transgender and nontrans patients do not receive statistically different quality of primary care. Trans patients' high engagement in primary care may result from providing hormone therapy and primary care within a single provider visit.
\end{abstract}

Keywords: HIV; primary care; primary care quality indicator; transgender

\section{Introduction}

Washington, DC, has the highest prevalence of transgender persons of any U.S. state at $2.8 \%{ }^{1}$; this is also greater than the estimated $0.3-0.5 \%$ worldwide prevalence. ${ }^{2}$ Self-administered surveys of DC's trans community reveal less education, lower income, and less stable housing coupled with greater HIV infection, compared to DC's nontrans population. ${ }^{3}$ However, data are lacking on primary care engagement and quality of care among transgender patients. ${ }^{4}$
Transgender persons face significant disparities in access to primary health care, due, in part, to lack of appropriate and competent medical care. ${ }^{3}$ A recent analysis of peer-reviewed transgender health literature from 2008 through 2014 found most studies addressed mental health, sexual health, or substance abuse, while barely $7 \%$ of articles focused on general health, including mortality, hormone use, and cancer; researchers concluded that "the general health of transgender people is the least researched aspect of the transgender

\footnotetext{
${ }^{1}$ Whitman-Walker Institute, Washington, District of Columbia.

${ }^{2}$ Division of Infectious Diseases, Department of Medicine, Georgetown University Medical Center, Washington, District of Columbia.

${ }^{3}$ Division of Infectious Diseases, Johns Hopkins University School of Medicine, Baltimore, Maryland.

*Address correspondence to: Deborah A. Goldstein, MD, Whitman-Walker Institute, 1525 14th Street, NW, Washington, DC 20005, E-mail: dgoldstein@whitman-walker.org Dr. Goldstein presented the data contained in this article at the GLMA annual conference in Philadelphia, PA, in September, 2017.
}

(C) Deborah A. Goldstein et al. 2019; Published by Mary Ann Liebert, Inc. This Open Access article is distributed under the terms of the Creative Commons License (http://creativecommons.org/licenses/by/4.0), which permits unrestricted use, distribution, and reproduction in any medium, provided the original work is properly cited. 
global burden of disease." ${ }^{2}$ A second review found no study addressing mammography, colorectal screenings, or influenza vaccinations, and suggested "an urgent need for research that addresses the primary care needs of all transgender and gender-nonconforming people."

Our motivation is to provide high-quality primary care to transgender patients. Because primary health care of transgender patients is an understudied area with few evidence-based guidelines, we analyzed primary care indicators from a large transgender cohort at Whitman-Walker Health (WWH), an HIV- and LGBT-focused Federally Qualified Health Center in Washington, DC. WWH has offered medical and support services for the trans community since 2005. In 2016, WWH served 8990 unique medical clients, including 1229 transgender (13.6\%) and 3285 (37\%) HIV-positive patients.

\section{Methods}

We conducted a retrospective electronic medical record (EMR) review between January 1, 2008 and January 1,2017 . We included patients $\geq 18$ years of age who self-identified as transgender on their initial clinic registration form, and who had attended at least one medical visit during the study period at either of WWH's two clinical sites in Washington, DC. WWH's research protocol for a retrospective trans health study was approved by Chespeake IRB in April 2016. This proposal was subsequently modified in November 2016 to broaden study population, dates of analysis, and include primary care quality indicators; this revised proposal was exempted from IRB oversight in December 2016.

WWH's clinic registration form queries transgender identity, but does not elicit specific information about natal sex and current gender identity; transgender identity is not stratified by trans female or trans male. For purposes of analysis, we defined trans female as someone assigned male sex at birth, whose current gender identity is female; trans male as someone assigned female sex at birth, whose current gender identity is male $^{2}$; and nonbinary person as someone who does not identify as trans female or trans male. WWH's EMR documents self-identified transgender patients with legal sex (sex on drivers license or insurance card) followed by "(T)." However, this practice may list gender identity or natal sex instead of legal sex. To create our transgender cohort, we identified all patients with " $(\mathrm{T})$ " in their chart and sorted by hormone use. Those with " $(\mathrm{T})$ " who were prescribed estrogens and/or antiandrogens were considered trans female; those with " $(\mathrm{T})$ " who were prescribed testosterones were considered trans male. We performed chart review for patients with "(T)," who were not prescribed hormone therapy and, based on providers' narrative charting, grouped these patients as trans female, trans male, or nonbinary. Nonbinary patients $(n=10)$ were excluded from the analysis.

We evaluated demographic factors, HIV infection, vaccinations, laboratory values, medical visits, and cancer screening among trans and nontrans cohorts, as well as gender-affirming hormone prescriptions and surgical referrals. Self-reported gender identity, race, ethnicity, and housing status from initial clinic registration forms were entered into eClinicalWorks (eCW), and then abstracted using BridgeIT software; SPSS was used for analysis. Age, insurance status at first appointment, residency at last appointment, and appointment data were handled similarly. Primary care indicators of interest were as follows: (1) at least one influenza vaccine during study period; (2) tobacco screening at first medical visit; (3) documentation of current smoking status; (4) at least one lipid screening during study period; (5) referral for mammogram; (6) referral for colonoscopy; and (7) cervical PAP screening. Primary care indicators were abstracted using ICD9, ICD10, and CPT codes, laboratory results, and external referrals. ${ }^{5}$ HIV status was determined by ICD9 and ICD10 codes for HIV infection; HIV status was reported as "unknown" if there were no ICD9 or ICD10 codes for HIV infection and no HIV testing reported in eCW. eCW was queried for medication prescriptions for hormones and gender-affirming surgical referrals, with a focus on medical indication and insurance type. We analyzed mean number of medical appointments during the study period as well as primary care engagement, defined as three or more appointments during the study period.

There are no transgender-specific primary care guidelines. The World Professional Association for Transgender Health ${ }^{6}$ recommends using country-specific guidelines where possible. We utilized recommendations from the University of California, San Francisco Center of Excellence for Transgender Health's Guidelines for the Primary and Gender-Affirming Care of Transgender and Gender Nonbinary People for tobacco screening, colonoscopy, and mammogram recommendations. ${ }^{7}$ We referenced U.S. Preventive Services Task Force (USPSTF) guidelines and WWH's internal 
protocol for transgender health care for lipid screening. ${ }^{8}$ We utilized USPSTF guidelines for cervical PAP screening ${ }^{9}$ and the 2016 Advisory Committee on Immunization Practices guidelines for influenza vaccination recommendations. ${ }^{10}$

\section{Statistical analyses}

Chi-square analysis was performed between gender identity and most primary care indicators. To better understand the effects of sociodemographic variables on primary care outcomes, the relationship between race, ethnicity, residency at last appointment, and insurance at first appointment and primary care indicators within each gender identity was examined using chi-square. Post hoc testing using adjusted standardized residuals was also performed. An analysis of variance (ANOVA) was performed between gender identity and mean medical appointments. An alpha level of 0.05 was used for all statistical tests. Outcomes were compared to national Healthy People 2020 primary care benchmarks. ${ }^{11}$

\section{Results}

Demographics

Of 20,097 patients seen during 2008-2016, 1822 were transgender $(9.0 \%)$, with $677(37.2 \%)$ trans males and 1145 (62.9\%) trans females; 18,275 were nontransgender (Table 1). Annually, the number of transgender patients receiving care at $\mathrm{WWH}$, defined as at least one medical visit in a calendar year, increased each year during the study period, with 790 trans females and 439 trans males in care in 2016 (Fig. 1). Among trans females, 31.3\% identified as African American; 50.8\% were between age $25-40 ; 20.2 \%$ identified as Hispanic or Latina; $20.1 \%$ reported unstable or temporary housing; and $28.1 \%$ had public insurance.

Bivariate analysis demonstrated a statistically significant association between age, race, insurance status at first appointment, housing status at first appointment, and gender identity. Trans males are more likely to be between age $18-24(p<0.001)$; white $(p<0.001)$; and privately insured $(p<0.001)$; and have stable/permanent housing at their first medical appointment $(p<0.001)$ compared to trans females and nontrans patients (Table 1). While most of WWHs nontrans population resided within $\mathrm{DC}$, the majority of trans patients lived outside of Washington, DC (75.5\% nontrans, vs. $47.8 \%$ trans females and 30.3\% trans males), with many traveling from West Virginia, New York, Pennsylvania, North Carolina, Georgia, and Florida (Table 1).
HIV

Despite high HIV infection rates among trans females, the nontrans population was more likely to be HIV positive when compared to both trans male and trans female patients $(p<0.001)$. Trans males were more likely to have unknown HIV status, indicating an absence of HIV testing at WWH during the study period (59.1\% of trans males vs. $28.0 \%$ of trans females and $34.6 \%$ of nontrans) (Table 1 ).

\section{Gender-affirming care}

More than $90 \%$ of trans patients received prescriptions for gender-affirming hormone therapy $(94.2 \%$ trans females and $91.3 \%$ trans males) (Table 2). Of trans females who were prescribed estrogen, $95.7 \%$ received estrogen with an antiandrogen and $4.3 \%$ received estrogen alone. All trans males on hormone therapy were prescribed testosterone. WWH's protocol for genderaffirming hormone therapy was previously reported. ${ }^{12}$

The proportion of transgender patients receiving surgical referrals for gender-affirming care increased from 2014 to 2016: in 2014, 4.5\% received surgical referrals, followed by $11.4 \%$ in 2015 and $18.7 \%$ in 2016 . In 2016, among the 230 trans patients who received surgical referrals for gender-affirming care, $67.8 \%$ had Medicaid, $24.3 \%$ had private insurance, 5.2\% had Medicare, and $2.6 \%$ were self-pay. The most common indications for surgical referrals in 2016 were breast augmentation and facial feminization for trans females, and mastectomy and hysterectomy for trans males (Fig. 2).

An ANOVA showed a statistically significant difference in mean medical appointments among the three gender identities $[F=(2,20094)=12.93, p<0.001$; Table 3]. A Tamhane post hoc test suggested that the mean number of medical appointments during the study period for nontrans patients (mean $=9.64$, standard deviation $[S D]=12.9)$ was significantly lower than the mean number of medical appointments for trans female patients (mean $=11.38, \mathrm{SD}=10.8 ; p<0.001$ ), and mean number of medical appointments for trans males (mean $=8.57, \mathrm{SD}=7.4$ ) was significantly lower compared with both trans females $(p<0.001)$ and nontrans patients $(p=0.001)$. Trans female patients had greater engagement in primary care, defined as more than three medical appointments during the study period, than trans male and nontrans patients $(p<0.001)$.

\section{Primary care indicators}

Trans males were less likely to receive at least one influenza vaccine than trans females or nontrans patients 
Table 1. Demographics of Transgender and Nontransgender Patients at Whitman-Walker Health, January 2008 to January 2017

\begin{tabular}{|c|c|c|c|c|c|c|c|}
\hline \multirow[b]{3}{*}{ Total } & \multicolumn{2}{|c|}{ Transgender females } & \multicolumn{2}{|c|}{ Transgender males } & \multicolumn{2}{|c|}{ Nontrans patients } & \multirow[b]{3}{*}{$p^{\mathrm{a}}$} \\
\hline & $n$ & $\%$ & $n$ & $\%$ & $n$ & $\%$ & \\
\hline & 1145 & 100.0 & 677 & 100.0 & 18275 & 100.0 & \\
\hline Age at first appointment & & & & & & & $<0.001$ \\
\hline $18-24$ & 375 & 32.8 & 332 & 49.0 & 2486 & 13.6 & \\
\hline $25-40$ & 582 & 50.8 & 291 & 43.0 & 8802 & 48.2 & \\
\hline $41-65$ & 187 & 16.3 & 53 & 7.8 & 6614 & 36.2 & \\
\hline$>65$ & 1 & 0.1 & 1 & 0.1 & 373 & 2.0 & \\
\hline Race & & & & & & & $<0.001$ \\
\hline White & 532 & 46.5 & 424 & 62.6 & 7070 & 38.7 & \\
\hline Black/African American & 356 & 31.1 & 137 & 20.2 & 7644 & 41.8 & \\
\hline Asian & 38 & 3.3 & 19 & 2.8 & 573 & 3.1 & \\
\hline Other & 46 & 4.0 & 29 & 4.3 & 355 & 1.9 & \\
\hline Unreported & 173 & 15.1 & 68 & 10.0 & 2633 & 14.4 & \\
\hline Ethnicity & & & & & & & $<0.001$ \\
\hline Hispanic or Latino & 256 & 22.4 & 49 & 7.2 & 3071 & 16.8 & \\
\hline Not Hispanic or Latino & 781 & 68.2 & 551 & 81.4 & 13465 & 73.7 & \\
\hline Unreported & 108 & 9.4 & 77 & 11.4 & 1739 & 9.5 & \\
\hline Housing status at first appointment & & & & & & & $<0.001$ \\
\hline Stable/permanent & 777 & 67.9 & 515 & 76.1 & 11484 & 62.8 & \\
\hline Unstable/temporary & 230 & 20.1 & 99 & 14.6 & 2436 & 13.3 & \\
\hline Unreported & 138 & 12.1 & 63 & 9.3 & 4355 & 23.8 & \\
\hline Insurance at first appointment ${ }^{\mathrm{b}}$ & & & & & & & $<0.001$ \\
\hline Private & 432 & 38.6 & 420 & 63.2 & 6419 & 37.4 & \\
\hline Public & 314 & 28.1 & 60 & 9.0 & 6693 & 39.0 & \\
\hline Self-pay & 231 & 20.6 & 128 & 19.2 & 2406 & 14.0 & \\
\hline Other & 142 & 12.7 & 57 & 8.6 & 1636 & 9.5 & \\
\hline Residency at last appointment, state & & & & & & & - \\
\hline District of Columbia & 547 & 47.8 & 205 & 30.3 & 13790 & 75.5 & \\
\hline Maryland & 247 & 21.6 & 217 & 32.1 & 2275 & 12.4 & \\
\hline Virginia & 314 & 27.4 & 223 & 32.9 & 1906 & 10.4 & \\
\hline Other Southern states ${ }^{c}$ & 18 & 1.6 & 19 & 2.8 & 102 & 0.6 & \\
\hline Northeast $^{\mathrm{d}}$ & 9 & 0.8 & 7 & 1.0 & 76 & 0.4 & \\
\hline Other states ${ }^{\mathrm{e}}$ & 10 & 0.9 & 6 & 0.9 & 88 & 0.5 & \\
\hline No data available & 0 & 0.0 & 0 & 0.0 & 38 & 0.2 & \\
\hline HIV status & & & & & & & $<0.001$ \\
\hline Positive $^{f}$ & 305 & 26.6 & 7 & 1.0 & 6494 & 35.5 & \\
\hline Negative HIV test & 519 & 45.3 & 270 & 39.9 & 5454 & 29.8 & \\
\hline Unknown status & 321 & 28.0 & 400 & 59.1 & 6327 & 34.6 & \\
\hline
\end{tabular}

${ }^{\mathrm{a}} \mathrm{A}$ chi-square test of independence at an alpha level of 0.05 was used to obtain all $p$-values reported.

${ }^{\mathrm{b}}$ Reporting insurance status for $97.7 \%$ of trans females, $98.2 \%$ of trans males, and $93.9 \%$ of nontrans patients. Data missing for 1115 participants. 'Other Southern states include the following: DE, $n=2 ; \mathrm{WV}, n=32 ; \mathrm{KY}, n=5 ; \mathrm{TN}, n=3 ; \mathrm{NC}, n=17 ; \mathrm{SC}, n=5 ; \mathrm{GA}, n=17 ; \mathrm{FL}, n=26 ; \mathrm{AL}, n=2 ; \mathrm{MS}, n=1$; $\mathrm{LA}, n=2 ; \mathrm{OK}, n=1$; and TX, $n=14$ (totals for entire cohort in each state).

${ }^{\mathrm{d}}$ Northeastern states include the following: $\mathrm{PA}, n=32 ; \mathrm{NJ}, n=9 ; \mathrm{CT}, n=6 ; \mathrm{MA}, n=9 ; \mathrm{NY}, n=35$; and VT, $n=2$ (totals for entire cohort in each state). 'Other states include the following: SD, $n=1 ; \mathrm{KS}, n=1 ; \mathrm{MN}, n=1 ; \mathrm{IA}, n=2 ; \mathrm{MO}, n=6 ; \mathrm{Wl}, n=3 ; \mathrm{IL}, n=11 ; \mathrm{IN}, n=1 ; \mathrm{Ml}, n=3 ; \mathrm{OH}, n=3 ; \mathrm{WA}, n=8 ; \mathrm{OR}$, $n=2 ; \mathrm{CA}, n=28 ; \mathrm{AK}, n=1 ; \mathrm{ID}, n=1 ; \mathrm{NV}, n=3 ; \mathrm{MT}, n=1 ; \mathrm{UT}, n=2 ; \mathrm{AZ}, n=2 ; \mathrm{CO}, n=6 ; \mathrm{NM}, n=1 ;$ and $\mathrm{HI}, n=1$; and international countries (totals for entire cohort in each state).

${ }^{f}$ Number of subjects who were ever HIV positive during study period, based on ICD-9 and ICD-10 codes and results of HIV tests in WWHs EMR. Unknown status indicates the absence of HIV testing at WWH during the study period.

EMR, electronic medical record; WWH, Whitman-Walker Health.

$(p<0.001 ;$ Table 3). Trans males were more likely to receive tobacco screening at their first medical visit $(p<0.001)$. Nontrans patients were more likely to identify as current smokers $(p<0.001)$. Trans male patients were more likely to receive at least one lipid screening than trans females and nontrans patients $(p<0.001)$. There was no statistically significant association between gender identity and either mammogram referrals $(p=0.107)$, or colonoscopy referrals $(p=0.683)$. There is a statistically significant association between cervical pap screening and gender identity, with trans males more likely to have had at least one cervical pap smear than cis females $(p=0.001)$. All three groups scored lower than the goals set forth by HealthyPeople2020 for influenza vaccination, mammogram referral, colonoscopy referral, and cervical pap smears ${ }^{11}$ (Table 3 ). 


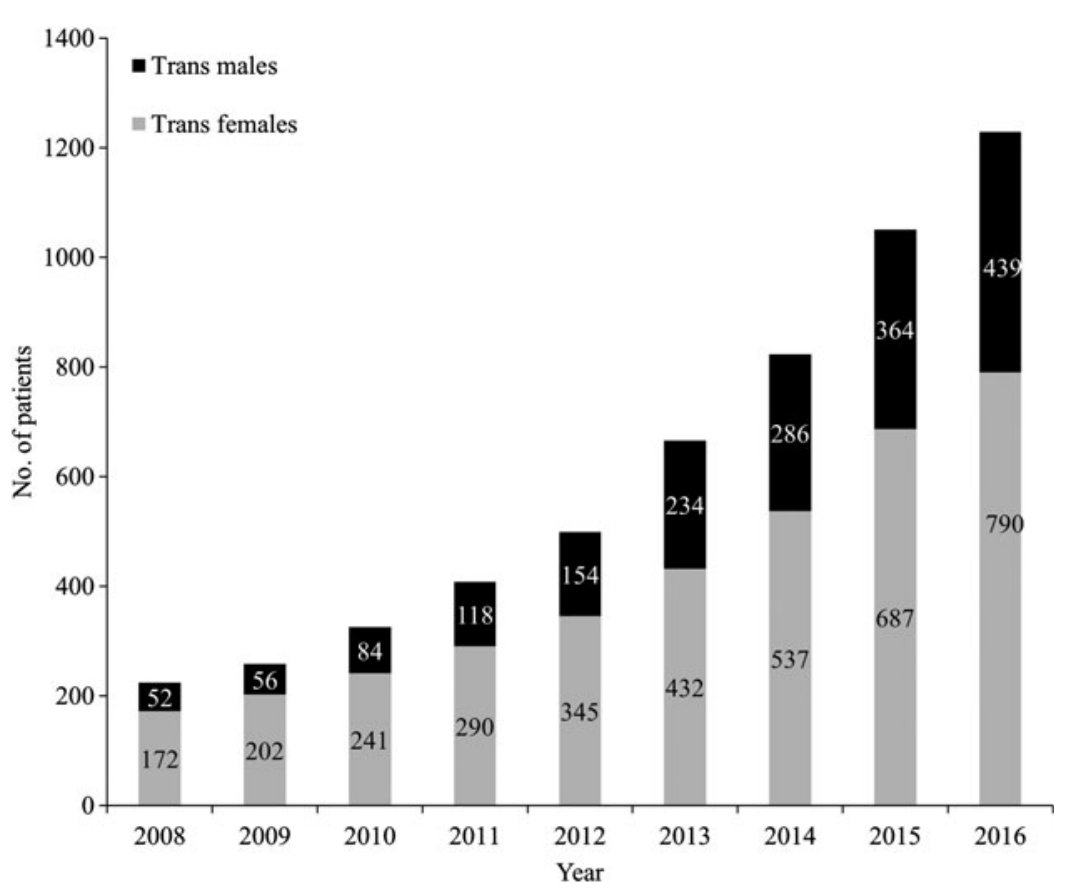

FIG. 1. Transgender patients in medical care at WWH by year, 2008-2016. "In medical care" is defined as at least one medical appointment with a WWH provider in a specific calendar year. WWH, Whitman-Walker Health.

Post hoc analysis of socioeconomic variables among trans females showed: (1) influenza vaccination was greater than expected among African Americans, DC residents, and publicly insured; (2) identification as smokers was greater than expected among African Americans and DC residents; and (3) receipt of at least one lipid screening and referrals for mammogram and colonoscopy were greater than expected among DC residents (Table 4). Among trans males, receipt of at least one cervical Pap smear was greater than expected among DC residents.

\section{Discussion}

This is the largest single-center US transgender cohort presented to date, and also the first medical care quality

Table 2. Hormone Use by Transgender Patients at Whitman-Walker Health, January 2008 to January 2017

\begin{tabular}{lcc}
\hline Transgender females & $n=1145$ & $100 \%$ \\
Estrogen and antiandrogen & 1014 & 88.6 \\
Estrogen alone & 46 & 4.0 \\
Antiandrogens alone & 18 & 1.6 \\
No hormone therapy & 67 & 5.8 \\
Transgender males & $n=677$ & $100 \%$ \\
Testosterone therapy & 618 & 91.3 \\
No hormone therapy & 59 & 8.7 \\
\hline
\end{tabular}

study of mammography, colorectal cancer screening, and influenza vaccination in transgender persons, to the best of our knowledge. ${ }^{13,14}$ This cohort is unique because it is predominantly trans female, privately insured, and has a high proportion of trans females with HIV infection, unlike other well-characterized US transgender cohorts. ${ }^{13,15}$ Compared to WWH's nontrans patients, transgender patients at $\mathrm{WWH}$ are more likely to be young, white, privately insured, have stable/permanent housing, and reside outside of Washington, DC. Trans females are more likely to identify as African American and/or Hispanic/Latina and have unstable/temporary housing and public health insurance, while trans males are more likely to identify as white, have stable/permanent housing, and have private health insurance.

\section{Gender-affirming care}

WWH's transgender care model envisions genderaffirming care, including hormone therapy and surgical referrals for gender transition, as an important part of primary care; therefore, WWH's medical providers integrate transgender care into primary care appointments. We hypothesize that transgender patients' high degree of primary care engagement is a reflection 


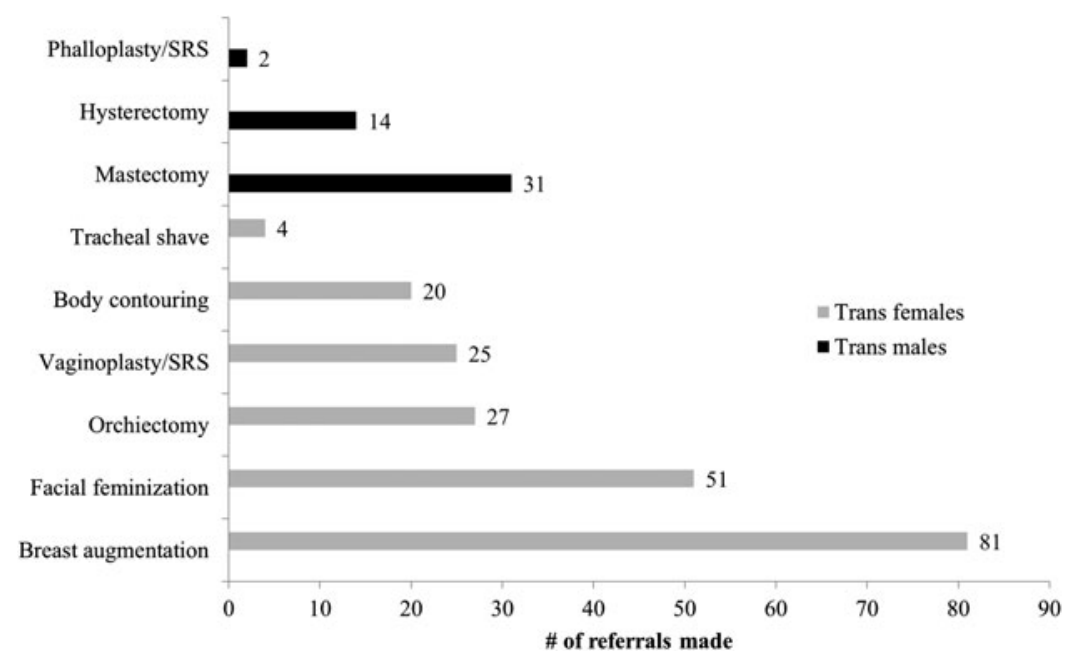

FIG. 2. Surgical referrals by indication for gender-affirming care of transgender patients, WWH, 2016.

Table 3. Primary Care Indicators for Transgender Patients at Whitman-Walker Health, January 2008 to January 2017

\begin{tabular}{|c|c|c|c|c|c|c|c|c|}
\hline \multirow[b]{3}{*}{ Total } & \multicolumn{2}{|c|}{ Transgender females } & \multicolumn{2}{|c|}{ Transgender males } & \multicolumn{2}{|c|}{ Nontrans patients } & \multirow[b]{3}{*}{$p^{\mathrm{a}}$} & \multirow{3}{*}{$\begin{array}{c}\text { Healthy People } \\
2020(\%)\end{array}$} \\
\hline & $n$ & $\%$ & $n$ & $\%$ & $n$ & $\%$ & & \\
\hline & 1145 & $100.0 \%$ & 677 & $100.0 \%$ & 18275 & $100.0 \%$ & & \\
\hline \multirow{2}{*}{$\begin{array}{l}\text { Mean, medical appointments } \\
\text { Primary care engagement } \\
>3 \text { Appointments }\end{array}$} & \multicolumn{2}{|c|}{$11.4(\mathrm{SD}=10.8)$} & \multicolumn{2}{|c|}{$8.6(\mathrm{SD}=7.4)$} & \multicolumn{2}{|c|}{$9.6(\mathrm{SD}=12.9)$} & $<0.001$ & \\
\hline & & & 74.0 & 10209 & 55.9 & $<0.001$ & \\
\hline Influenza vaccinations & & & & & & & $<0.001$ & 80.0 \\
\hline At least one vaccination & 451 & 39.4 & 161 & 23.8 & 6918 & 37.9 & & \\
\hline Never vaccinated & 694 & 60.6 & 516 & 76.2 & 11357 & 62.1 & & \\
\hline Tobacco screen at baseline & & & & & & & $<0.001$ & 68.6 \\
\hline Screened at baseline & 999 & 87.2 & 604 & 89.2 & 12030 & 65.8 & & \\
\hline Never screened & 146 & 12.8 & 73 & 10.8 & 6245 & 34.2 & & \\
\hline Smoking status at baseline ${ }^{b}$ & & & & & & & $<0.001$ & \\
\hline Current smoker & 250 & 25.0 & 128 & 21.2 & 3471 & 28.9 & & \\
\hline Nonsmoker & 749 & 75.0 & 476 & 78.8 & 8559 & 71.1 & & \\
\hline Lipid screenings ${ }^{c}$ & & & & & & & $<0.001$ & 82.1 \\
\hline At least one screen & 826 & 74.4 & 539 & 86.1 & 11617 & 64.0 & & \\
\hline Never screened & 284 & 25.6 & 87 & 13.9 & 6528 & 36.0 & & \\
\hline Mammogram referrals ${ }^{d}$ & & & & & & & 0.107 & 81.1 \\
\hline At least one referral & 37 & 33.9 & 4 & 21.1 & 653 & 40.2 & & \\
\hline Never referred & 72 & 66.1 & 15 & 78.9 & 970 & 59.8 & & \\
\hline Colonoscopy referrals $^{\mathrm{e}}$ & & & & & & & 0.683 & 70.5 \\
\hline At least one referral & 33 & 30.3 & 4 & 21.1 & 1372 & 30.3 & & \\
\hline Never referred & 76 & 69.7 & 15 & 78.9 & 3159 & 69.7 & & \\
\hline Cervical pap smears ${ }^{f}$ & & & & & & & $<0.001$ & 93.0 \\
\hline At least one pap smear & & & 337 & 56.2 & 3167 & 49.1 & & \\
\hline No pap smear & & & 263 & 43.8 & 3278 & 50.9 & & \\
\hline
\end{tabular}

${ }^{\mathrm{a}} \mathrm{A}$ chi-square test of independence at an alpha level of 0.05 was used to obtain all $p$-values reported, except for mean medical appointments. For mean medical appointments, the one-way ANOVA test was used to obtain the $p$-value.

${ }^{\mathrm{b}}$ Only includes patients with tobacco screen data.

'Only includes patients who were ever 20 years of age and older during the study period.

dOnly includes transgender female, transgender male, and cisgender female patients who were ever 50 years of age and older during the study period.

e Only includes patients who were ever 50 years of age and older during the study period.

fOnly includes transgender male and cisgender female patients who were ever 21 years of age and older during the study period.

ANOVA, analysis of variance; SD, standard deviation. 
Table 4. Adjusted Standardized Residuals for Associations Between Sociodemographic Factors and Primary Care Indicators by Gender Identity

\begin{tabular}{|c|c|c|c|c|c|c|c|c|}
\hline & \multicolumn{6}{|c|}{ Transgender females } & \multicolumn{2}{|c|}{ Transgender males ${ }^{a}$} \\
\hline & $\begin{array}{l}\text { Influenza } \\
\text { vaccine }\end{array}$ & $\begin{array}{l}\text { Tobacco } \\
\text { screen }\end{array}$ & $\begin{array}{l}\text { Smoking } \\
\text { status }\end{array}$ & $\begin{array}{l}\text { Lipid } \\
\text { screening }\end{array}$ & Mammograms & Colonoscopy & $\begin{array}{l}\text { Influenza } \\
\text { vaccine }\end{array}$ & $\begin{array}{c}\text { Cervical } \\
\text { pap smears }\end{array}$ \\
\hline \multicolumn{9}{|l|}{ Race } \\
\hline White & $166(-5.3)^{b}$ & $-^{c}$ & $58(-8.8)$ & - & - & - & - & $209(0.3)$ \\
\hline African American & $183(5.6)$ & - & $147(11)$ & - & - & - & - & $85(2.4)$ \\
\hline Asian & $13(-0.7)$ & - & $5(-1)$ & - & - & - & - & $4(-1.9)$ \\
\hline Other & $18(0)$ & - & $6(-1.7)$ & - & - & - & - & $10(-2)$ \\
\hline \multicolumn{9}{|l|}{ Ethnicity } \\
\hline Hispanic & - & $234(2.3)$ & - & $196(1.9)$ & - & - & - & - \\
\hline Non-Hispanic & - & $688(-2.6)$ & - & $566(0.3)$ & - & - & - & - \\
\hline \multicolumn{9}{|l|}{ Residency } \\
\hline DC resident & $271(6.8)$ & - & $177(8.2)$ & $426(4)$ & $24(2)$ & $23(2.5)$ & $59(2)$ & $135(4.2)$ \\
\hline Non-DC resident & $180(-6.8)$ & - & $73(-8.2)$ & $400(-4)$ & $13(-2)$ & $10(-2.5)$ & $102(-2)$ & $202(-4.2)$ \\
\hline \multicolumn{9}{|l|}{ Insurance } \\
\hline Private & $137(-4.1)$ & - & - & $289(-2.7)$ & - & - & - & - \\
\hline Public & $171(6.4)$ & - & - & $241(2.2)$ & - & - & - & - \\
\hline Self pay & $76(-2.3)$ & - & - & $164(-0.5)$ & - & - & - & - \\
\hline Other & $52(-0.7)$ & - & - & $110(1)$ & - & - & - & - \\
\hline
\end{tabular}

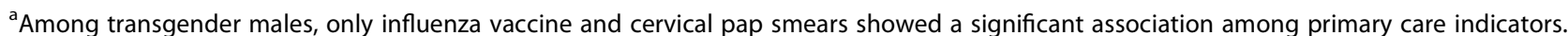

${ }^{b}$ Reported results are observed counts with adjusted standardized residuals in parentheses. Adjusted standardized residuals were considered significant if $>3$ or $<-3$.

'Observed counts and adjusted standardized residuals were only reported for associations that were statistically significant with a primary care outcome at $p<0.05$.

of this care model. More than $90 \%$ of WWH transgender patients received gender-affirming hormone therapy; similarly, Fenway Health reported $81 \%$ of trans males and $89 \%$ of trans females received hormonal therapy. ${ }^{15}$ The recent increase in referrals for genderaffirming surgeries is, in large part, due to an updated DC Medicaid policy for coverage of gender reassignment surgery. ${ }^{16}$ The increasing availability of surgical specialists in the Washington, DC, area also influenced this trend. ${ }^{17}$

\section{HIV testing}

The CDC recommends that persons at risk for HIV infection be screened for HIV at least annually, although transgender persons are not specifically discussed in current recommendations. ${ }^{18}$ Some studies have found that transgender patients undergo HIV screening at rates higher than the general population. ${ }^{4}$ However, in a recent survey of transgender respondents, trans females and trans males self-reported HIV testing at levels similar to cisgender heterosexuals, and at levels inconsistent with their HIV risk. ${ }^{18}$ Our data reveal a HIV testing deficit among trans males at WWH. This finding requires additional exploration of the unique testing barriers this population faces, including providers' pre-conceptions about sexual activity and HIV risk.

\section{HIV infection}

The $28 \%$ HIV seroprevalence among trans females is consistent with other published U.S. estimates of 1052.4\%. ${ }^{4}$ WWH specializes in providing HIV care, reflected by the large percentage of nontrans HIV+ persons in care. There are little available data regarding HIV seroprevalence among transgender males; the $1.0 \%$ HIV seropositivity among trans males in this study is consistent with previous reports of $0-11 \%$ HIV positivity. ${ }^{4}$ Many published reports of HIV seroprevalence among transgender persons are based on self-report, which likely underestimates seroprevalence. ${ }^{4}$ Results from the ongoing multi-site LITE study, a longitudinal cohort of HIV-negative trans females in the eastern and southern United States, will help to characterize risk factors for HIV infection and access to HIV prevention methods to inform evidence-based HIV prevention interventions. ${ }^{19}$

\section{Primary care indicators}

Annual influenza vaccination is recommended for all persons $\geq 6$ months of age without contraindications. ${ }^{10}$ Vaccination data among transgender patients are limited. In a recent report of LGBT respondents, $4 \%$ of whom identified as transgender, annual influenza vaccination prevalence reached $68.0 \% .{ }^{20}$ WWH's low rate of trans male influenza vaccination may be due 
to privately insured trans males traveling from outside Washington, DC, to receive gender-affirming care at WWH with primary care providers near home who administer influenza vaccinations; these vaccinations are not well captured by our EMR. Alternately, trans males, with low rates of tobacco use and HIV infection, may be perceived as low risk of influenza-related complications and not offered vaccination, or they may refuse vaccination.

Trans female smokers should be counseled on tobacco risks and cessation options at every visit; no specific recommendation is made for trans males. ${ }^{7}$ While previous studies have reported high rates of tobacco use among transgender persons, others comparing smoking rates among transgender people to cisgender lesbian, gay, and bisexual people did not find statistically significant higher rates of smoking. ${ }^{5}$ At $\mathrm{WWH}$, transgender patients were more likely to receive tobacco screening and less likely to identify as current smokers than nontrans patients. This may be because WWH, an LGBT-focused health center, has a relatively high rate of current smokers among nontrans patients, nearing $30 \%$. High lipid screening among trans females and trans males reflects WWH's clinical protocol, which includes baseline lipid panels before hormone initiation for all trans patients and a follow-up lipid panel for trans males 3 months after testosterone initiation. We evaluated lipid screening among those $\geq 20$ years of age during the study period, in keeping with the USPSTFs grade B recommendation. ${ }^{8}$

Clinical guidelines recommend trans females undergo screening mammography every 2 years beginning at the age of 50 and after receiving 5-10 years of feminizing hormones. ${ }^{7}$ Trans males who have not undergone bilateral mastectomy or who have only undergone breast reduction should undergo screening for cis females, although there is scant evidence to support this recommendation. ${ }^{7}$ Fenway Health reported transgender patients, including trans females and trans males without bilateral mastectomy, were less likely than cisgender females to adhere to mammography recommendations. $^{21}$ We restricted our analysis to those $\geq 50$ years of age during the study period; among trans females, we did not account for hormone duration, and among trans males we did not account for history of mastectomy or breast reduction, making it difficult to assess if mammogram referrals were consistent with published guidelines.

Growing literature suggests lower rates of cervical Pap tests among transgender males ${ }^{2}$ due to barriers to screening, including vaginal atrophy and dryness causing discomfort during examination. ${ }^{22}$ Fenway Health-reported trans males were less likely to be up to date on Pap tests than nontrans females. ${ }^{21}$ Our higher trans male Pap rate may reflect provider commitment to screen this population and secure documentation of Paps from outside providers. WWH Pap rates among both trans males and cis females are considerably lower than the HealthyPeople2020 benchmark and represent future areas for improvement.

DC residents' improved primary care outcomes may be due to broad availability of DC Medicaid or to underdocumentation of primary care interventions performed on non-DC-resident patients by health care providers outside of WWH.

\section{Limitations}

This retrospective EMR review is subject to bias from convenience sampling of our clinic population; inadequacy in capturing health data outside of our health system; and limitations of an EMR designed for a cisgender population. Our transgender cohort's predominant composition of young, white, HIV-negative, and privately insured patients suggests access to care not reported in previous transgender cohorts, and this impacts evaluation of primary care outcomes. It is difficult to capture the dynamic process of gender transition in a retrospective chart review. We did not match trans to cis gender patients, but instead compared transgender patients to a large mixed sample of nontrans patients. Our analysis of referrals for cancer screening instead of completion of screening is problematic and reflects the limitations of available EMR data; this methodology does not account for barriers in completing these examinations, including transphobic providers and fear of being misgendered. The underrepresentation of nonbinary patients is due to an insufficient method of identifying nonbinary patients in our EMR. HealthyPeople2020 indicators were developed for cisgender patients; their use as a guideline for transgender patients is problematic.

\section{Conclusion}

To establish comprehensive, evidence-based national guidelines for the primary care of transgender patients, additional research is needed to determine best practices. There is a need for prospective cohort studies to evaluate optimal age of initiation and optimal interval for repeating cancer screening in transgender patients on hormone therapy, including mammogram and cervical 
Pap smears. EMRs must strive to adequately document gender identity. Prospective interventions to increase HIV testing among trans males are imperative.

\section{Acknowledgments}

Chesapeake IRB determined this study to be exempt from IRB oversight in April 2017.

\section{Author Disclosure Statement}

No competing financial interests exist.

\section{Funding Information}

No funding was received for this article.

\section{References}

1. Flores AR, Herman JL, Gates GJ, Brown TN. How Many Adults Identify as Transgender in the United States? Los Angeles, CA: The Williams Institute.

2. Reisner SL, Poteat T, Keatley J, et al. Global health burden and needs of transgender populations: a review. Lancet. 2016;388:412-436.

3. Edelman EA, Corado R, Lumby EC, et al. Access denied: Washington, DC Trans Needs Assessment Report. DC Trans Coalition. 2015. Available at www.dctranscoalition.org Accessed March 2, 2017.

4. Edmiston KE, Donald CA, Sattler AR, et al. Opportunities and gaps in primary care preventative health services for transgender patients: a systematic review. Transgender Health. 2016;1.1:216-230.

5. AAPC Coder. Available at https://coder.aapc.com/search Accessed April 24, 2019.

6. The World Professional Association for Transgender Health. Standards of care for the health of transsexual, transgender, and gender nonconforming people, 7th version. Available at https://s3.amazonaws .com/amo_hub_content/Association140/files/Standards $\% 20$ of $\%$ 20Care\%20V7\%20-\%202011\%20WPATH\%20(2)(1).pdf Accessed October 5, 2017.

7. Deutsch MB. Guidelines for the primary and gender-affirming care of transgender and gender nonbinary people [Internet]. University of California, San Francisco, Center of Excellence for Transgender Health; 2016. Available at http://transhealth.ucsf.edu/trans?page=protocol-00-00 Accessed October 5, 2017.

8. The Guide to Clinical Preventive Services 2012: Recommendations of the U.S. Preventive Services Task Force, Rockville, MD: Agency for Healthcare Research and Quality; October 2012. Report No.: 12-05154.

9. Moyer VA; on behalf of the U.S. Preventive Services Task Force. Screening for cervical cancer: U.S. Preventive Services Task Force recommendation statement. Ann Intern Med. 2012;156:880-891.

10. Grohskopf LA, Sokoklow LZ, Broder KR, et al. Prevention and control of seasonal influenza with vaccines: recommendations of the Advisory Committee on Immunization Practices-United States, 2017-18 Influenza Season. MMWR Recomm Rep. 2017;66:1-24.

11. U.S. Department of Health and Human Services: Healthy People 2020 Leading Health Indicators. Available at www.healthypeople.gov/2020/ Leading-Health-Indicators Accessed October 4, 2017.

12. Arnold JD, Sarkodie EP, Coleman ME, et al. Incidence of venous thromboembolism in transgender women receiving oral estradiol. J Sex Med. 2016;13:1773-1777.

13. Brown GR, Jones KT. Mental health and medical health disparities in 5135 transgender veterans receiving healthcare in the Veterans Health Administration: a case-control study. LGBT Health. 2016;3:122-131.

14. Herbst JH, Jacobs ED, Finlayson TJ, et al. Estimating HIV prevalence and risk behaviors of transgender persons in the United States: a systematic review. AIDS Behav. 2008;12:1-17.

15. Reisner SL, Bradford J, Hopwood R, et al. Comprehensive transgender healthcare: the gender affirming clinical and public health model of Fenway Health. J Urban Health 2015;92:584-592.

16. Government of the District of Columbia, Department of Health Care Finance: Gender Reassignment Surgery Policy. Policy\#: OD-001-17.
Available at https://dhcf.dc.gov/sites/default/files/dc/sites/dhcf/ release_content/attachments/Policy\%20\%23\%200D-00117_Gender\%20Reassignment\%20Surgery.pdf Accessed April 26, 2018.

17. Arnold JD, Nelson AE, Loubier EM. Transgender surgery-not the benchmark for gender marker determination. JAMA Surg. 2017;152:1099-1100.

18. Pitasi MA, Oraka $\mathrm{E}$, Clark $\mathrm{H}$, et al. HIV testing among transgender women and men-27 States and Guam, 2014-2015. MMWR Morb Mortal Wkly Rep. 2017;66:883-887.

19. Leading Innovation for Transgender Women's Health and Empowerment: The LITE Study. Available at https://www.litestudy.org Accessed June 13, 2018.

20. Jones J, Poole A, Lasley-Bibbs V, et al. LGBT health and vaccinations: findings from a community health survey of Lexington-Fayette County, Kentucky, USA. Vaccine. 2016;34:1909-1914.

21. Bazzi AR, Whorms DS, King DS, et al. Adherence to mammography screening guidelines among transgender persons and sexual minority women. J Public Health. 2015;105:2356-2358.

22. Peitzmeier SM, Khullar K, Reisner SL, et al. Pap test use is lower among female-to-male patients than non-transgender women. Am J Prev Med. 2014:47:808-812.

Cite this article as: Goldstein DA, Sarkodie E, Hardy WD (2019) Transgender and nontrans patients do not receive statistically different quality primary care at Whitman-Walker Health, 2008-2016,

Transgender Health 4:1, 200-208, DOI: 10.1089/trgh.2019.0022.

$\begin{aligned} & \text { Abbreviations Used } \\ \mathrm{ANOVA} & =\text { analysis of variance } \\ \mathrm{eCW} & =\text { eClinicalWorks } \\ \mathrm{EMR} & =\text { electronic medical record } \\ \mathrm{SD} & =\text { standard deviation } \\ \mathrm{USPSTF} & =\text { U.S. Preventive Services Task Force } \\ \mathrm{WWH} & =\text { Whitman-Walker Health }\end{aligned}$

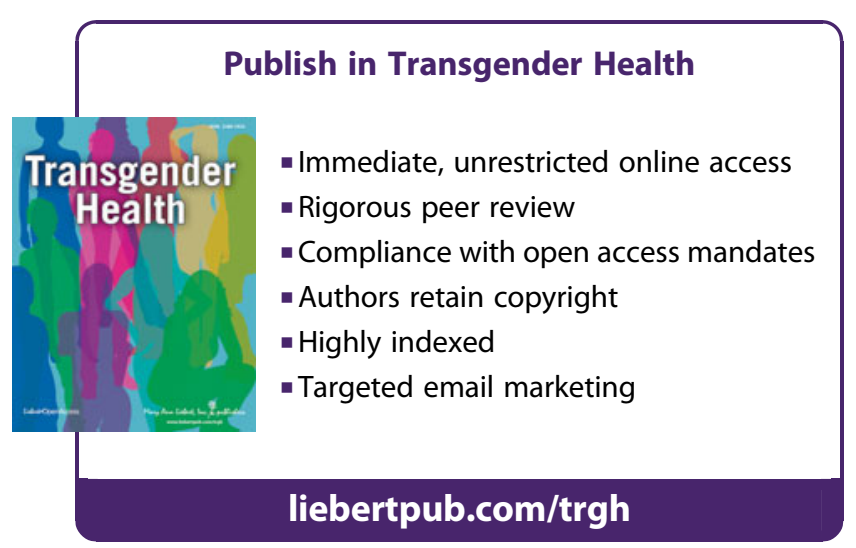

